\title{
A CHAIN RULE FOR MULTIVARIABLE RESULTANTS
}

\author{
CHARLES CHING-AN CHENG, JAMES H. MCKAY, AND STUART SUI-SHENG WANG
}

(Communicated by Maurice Auslander)

\begin{abstract}
We present a chain rule for the multivariable resultant, which is similar to the familiar chain rule for the Jacobian matrix. Specifically, given two homogeneous polynomial maps $K^{n} \rightarrow K^{n}$ for a commutative ring $K$, such that their composition is a homogeneous polynomial map, the resultant of the composition is the product of appropriate powers of resultants of the individual maps.
\end{abstract}

The chain rule for Jacobians is well known. There are some similarities between Jacobians and resultants. In [11], [12] and [13], the chain rule for resultants is proven for two polynomials in one variable as well as two homogeneous polynomials in two variables. (In [7], the chain rule is also proven for two homogeneous polynomials in two variables.) In this paper, a chain rule for the multivariable resultant is presented which generalizes the chain rule for resultants to $n$ variables. The result is "universal" because the polynomials have indeterminate coefficients. The proof is more "conceptual" since it is based on the four axioms characterizing the multivariable resultant.

In Section 1 the main result is stated and proved. In Section 2 consequences of the main result are derived; these include generalizations of the previous work on the chain rule of resultants. In Section 3 an axiomatic characterization of the multivariable resultant is included for the convenience of the reader.

\section{The MAIN ReSUlT}

A set of polynomials is said to be generic if the polynomials are complete in all their terms with (independent) indeterminate coefficients.

Theorem 1 (Chain rule for generic homogeneous polynomials). Suppose

$$
F_{1}\left(x_{1}, \ldots, x_{n}\right), \ldots, F_{n}\left(x_{1}, \ldots, x_{n}\right), H_{1}\left(u_{1}, \ldots, u_{n}\right), \ldots, H_{n}\left(u_{1}, \ldots, u_{n}\right)
$$

are generic homogeneous polynomials of positive (total) degrees $l_{1}, \ldots, l_{n}$,

Received by the editors March 23, 1992 and, in revised form, July 16, 1993.

1991 Mathematics Subject Classification. Primary 16A72, 13A50, 13B25, 12D10, 12 E05.

Key words and phrases. Multivariable resultant, common zeros, generic polynomials, Jacobian Conjecture, chain rule, Nullstellensatz, discriminant, isobaric property, invariant. 
$d, \ldots, d$. Then

$$
\begin{aligned}
& \operatorname{Res}_{u_{1}, \ldots, u_{n}}\left(F_{1}\left(H_{1}, \ldots, H_{n}\right), \ldots, F_{n}\left(H_{1}, \ldots, H_{n}\right)\right) \\
& \quad=\left[\operatorname{Res}_{x_{1}, \ldots, x_{n}}\left(F_{1}, \ldots, F_{n}\right)\right]^{d^{n-1}}\left[\operatorname{Res}_{u_{1}, \ldots, u_{n}}\left(H_{1}, \ldots, H_{n}\right)\right]^{l_{1} \cdots l_{n}}
\end{aligned}
$$

where the equality holds in the polynomial ring $\mathbb{Z}\left[\right.$ coefficients of $F_{1}, \ldots, F_{n}$, $\left.\left.H_{1}, \ldots, H n\right\}\right]$.

Proof. To simplify the notation, let

$$
\begin{aligned}
C_{i}\left(u_{1}, \ldots, u_{n}\right) & =F_{i}\left(H_{1}, \ldots, H_{n}\right) \quad \text { for } i=1,2, \ldots, n, \\
\mathrm{R}_{\mathrm{c}} & =\operatorname{Res}_{u_{1}, \ldots, u_{n}}\left(C_{1}, C_{2}, \ldots, C_{n}\right), \\
\mathrm{R}_{\mathrm{F}} & =\operatorname{Res}_{x_{1}, \ldots, x_{n}}\left(F_{1}, F_{2}, \ldots, F_{n}\right), \\
\mathrm{R}_{\mathrm{H}} & =\operatorname{Res}_{u_{1}, \ldots, u_{n}}\left(H_{1}, H_{2}, \ldots, H_{n}\right) .
\end{aligned}
$$

We need to show that $\mathrm{R}_{\mathrm{C}}=\mathrm{R}_{\mathrm{F}}^{d^{n-1}} \mathrm{R}_{\mathrm{H}}{ }^{l_{1} l_{2} \cdots l_{n}}$.

Note that

$$
\begin{aligned}
\mathbf{R}_{\mathrm{C}}, \mathbf{R}_{\mathrm{F}}, \mathbf{R}_{\mathrm{H}} & \in \mathbb{Z}\left[\left\{\text { coefficients of } F_{1}, \ldots, F_{n}, H_{1}, \ldots, H_{n}\right\}\right] \\
& \subset \mathbb{Q}\left[\left\{\text { coefficients of } F_{1}, \ldots, F_{n}, H_{1}, \ldots, H_{n}\right\}\right] \\
& \subset \mathbb{C}\left[\left\{\text { coefficients of } F_{1}, \ldots, F_{n}, H_{1}, \ldots, H_{n}\right\}\right] .
\end{aligned}
$$

We claim that

$$
R_{C} \text { vanishes whenever } R_{F} R_{H} \text { vanishes, and vice versa. }
$$

Using Hilbert's Nullstellensatz [1, Chapter V, §3.3, Proposition 2(iv), p. 351], this implies that $R_{C}$ and $R_{F} R_{H}$ have the same irreducible factors in the polynomial ring $\mathbb{Q}\left[\left\{\right.\right.$ coefficients of $\left.\left.F_{1}, \ldots, F_{n}, H_{1}, \ldots, H_{n}\right\}\right]$. Since both $\mathrm{R}_{\mathrm{F}}$ and $\mathbf{R}_{\mathrm{H}}$ are irreducible by (2) of Theorem 16, it follows that

$$
\mathbf{R}_{\mathrm{C}}=\lambda \mathbf{R}_{\mathrm{F}}^{a} \mathbf{R}_{\mathbf{H}}^{b}
$$

for some $\lambda \in \mathbb{Q}$ and some positive integers $a$ and $b$. Setting $F_{1}=a_{1} x_{1}^{l_{1}}$, $F_{2}=a_{2} x_{2}^{l_{2}}, \ldots, F_{n}=a_{n} x_{n}^{l_{n}}, H_{1}=b_{1} u_{1}^{d}, H_{2}=b_{2} u_{2}^{d}, \ldots, H_{n}=b_{n} u_{n}^{d}$ and using (4) of Theorem 16 yield

$$
\begin{aligned}
& \mathbf{R}_{\mathrm{C}}=\prod_{i=1}^{n}\left(a_{i} b_{i}^{l_{i}}\right)^{\left(d l_{1}\right) \cdots \widehat{\left(d l_{i}\right)} \cdots\left(d l_{n}\right)}, \\
& \mathbf{R}_{\mathrm{F}}=\prod_{i=1}^{n} a_{i}^{l_{1} \cdots \hat{l}_{i} \cdots l_{n}}, \\
& \mathbf{R}_{\mathrm{H}}=\prod_{i=1}^{n} b_{i}^{d^{n-1}}
\end{aligned}
$$

where $\hat{l}_{i}$ means that $l_{i}$ is omitted. Thus $a=d^{n-1}, b=l_{1} l_{2} \ldots l_{n}, \lambda=1$.

It remains to prove statement (2), i.e., for every ring homomorphism $\phi$ : $A \longrightarrow \mathbb{C}$,

$$
\phi\left(\mathbf{R}_{\mathrm{C}}\right)=0 \Longleftrightarrow \phi\left(\mathbf{R}_{\mathrm{F}}\right) \phi\left(\mathbf{R}_{\mathrm{H}}\right)=0
$$


where $A=\mathbb{Q}\left[\left\{\right.\right.$ coefficients of $\left.\left.F_{1}, \ldots, F_{n}, H_{1}, \ldots, H_{n}\right\}\right]$. For convenience we shall write $\bar{R}$ for $\phi(R)$ for all $R \in A$, and $\bar{P}$ for $\widetilde{\phi}(P)$ for all $P \in$ $A\left[x_{1}, \ldots, x_{n}, u_{1}, \ldots, u_{n}\right]$ where

$$
\tilde{\phi}: A\left[x_{1}, \ldots, x_{n}, u_{1}, \ldots, u_{n}\right] \longrightarrow \mathbb{C}\left[x_{1}, \ldots, x_{n}, u_{1}, \ldots, u_{n}\right]
$$

is the natural extension of $\phi$. Thus we need to establish that $\bar{R}_{\mathrm{C}}=0$ if and only if $\bar{R}_{\mathrm{F}} \overline{\mathrm{R}}_{\mathrm{H}}=0$. Using (1) of Theorem 16 and the fact that $\widetilde{\phi}$ distributes over composition, we have

$$
\begin{aligned}
& \overline{\mathrm{R}}_{\mathrm{C}}=\operatorname{Res}_{u_{1}, \ldots, u_{n}}\left(\bar{C}_{1}, \bar{C}_{2}, \ldots, \bar{C}_{n}\right), \\
& \overline{\mathrm{R}}_{\mathrm{F}}=\operatorname{Res}_{x_{1}, \ldots, x_{n}}\left(\bar{F}_{1}, \bar{F}_{2}, \ldots, \bar{F}_{n}\right), \\
& \overline{\mathbf{R}}_{\mathrm{H}}=\operatorname{Res}_{u_{1}, \ldots, u_{n}}\left(\bar{H}_{1}, \bar{H}_{2}, \ldots, \bar{H}_{n}\right) .
\end{aligned}
$$

If $\overline{\mathbf{R}}_{\mathrm{c}}=0$ then, by (3) of Theorem 16, there exists a nontrivial common zero $\left(\alpha_{1}, \ldots, \alpha_{n}\right) \in \mathbb{C}^{n}$ of $\bar{C}_{1}, \ldots, \bar{C}_{n}$. If it is also a common zero of $\bar{H}_{1}, \ldots, \bar{H}_{n}$, then $\overline{\mathbf{R}}_{\mathrm{H}}=0$. Otherwise, let $\beta_{i}=\bar{H}_{i}\left(\alpha_{1}, \ldots, \alpha_{n}\right)$ for each $i$; then $\left(\beta_{1}, \ldots, \beta_{n}\right)$ is a nontrivial common zero of $\bar{F}_{1}, \ldots, \bar{F}_{n}$ since $\bar{C}_{i}=$ $\bar{F}_{i}\left(\bar{H}_{1}, \ldots, \bar{H}_{n}\right)$ for all $i$ and so $\overline{\mathrm{R}}_{\mathrm{F}}=0$. Therefore $\overline{\mathrm{R}}_{\mathrm{c}}=0$ implies that $\overline{\mathbf{R}}_{\mathrm{H}}=0$ or $\overline{\mathbf{R}}_{\mathrm{F}}=0$.

If $\overline{\mathbf{R}}_{\mathrm{H}}=0$, then there exists a nontrivial common zero $\left(\alpha_{1}, \ldots, \alpha_{n}\right) \in \mathbb{C}^{n}$ of $\bar{H}_{1}, \ldots, \bar{H}_{n}$. Since $\bar{F}_{i}$ is either zero or homogeneous of positive degree $l_{i}, \bar{F}_{i}(0, \ldots, 0)=0$. Hence $\left(\alpha_{1}, \ldots, \alpha_{n}\right)$ is also a (nontrivial) common zero of $\bar{C}_{1}, \ldots, \bar{C}_{n}$. Therefore $\overline{\mathrm{R}}_{\mathrm{H}}=0$ implies $\overline{\mathrm{R}}_{\mathrm{C}}=0$.

If $\overline{\mathbf{R}}_{\mathrm{F}}=0$, then there exists a nontrivial common zero $\left(\beta_{1}, \ldots, \beta_{n}\right) \in \mathbb{C}^{n}$ of $\bar{F}_{1}, \ldots, \bar{F}_{n}$. By Lemma 3 , either the polynomials $\bar{H}_{1}-\beta_{1}, \ldots, \bar{H}_{n}-\beta_{n}$ have a nontrivial common zero $\left(\alpha_{1}, \ldots, \alpha_{n}\right) \in \mathbb{C}^{n}$ or the homogeneous polynomials $\bar{H}_{1}, \ldots, \bar{H}_{n}$ have a nontrivial common zero $\left(\alpha_{1}, \ldots, \alpha_{n}\right) \in \mathbb{C}^{n}$. In either case $\left(\alpha_{1}, \ldots, \alpha_{n}\right)$ is a nontrivial common zero of $\bar{C}_{1}, \ldots, \bar{C}_{n}$. Therefore $\overline{\mathbf{R}}_{\mathrm{H}}=0$ implies $\overline{\mathbf{R}}_{\mathrm{c}}=0$.

Lemma 2. Over an algebraically closed field, a system of homogeneous polynomial equations with more unknowns than equations has a nontrivial solution, provided that none of the polynomials is a nonzero constant.

Proof. This can be derived from the Krull's Principal Ideal Theorem (see, e.g., [8, Chapter V, Corollary 3.6, p. 133 and Chapter II, Corollary 3.8, p. 54].)

Lemma 3. Suppose, for each $i=1,2, \ldots, n, \bar{H}_{i} \in \mathbb{C}\left[u_{1}, \ldots, u_{n}\right]$ is either zero or homogeneous of positive degree $d$. Let $\left(\beta_{1}, \ldots, \beta_{n}\right) \neq(0, \ldots, 0)$ be an element of $\mathbb{C}^{n}$. Then there exists $\left(\alpha_{1}, \ldots, \alpha_{n}\right) \neq(0, \ldots, 0)$ in $\mathbb{C}^{n}$ such that

$$
\text { either } \quad\left\{\begin{array} { c } 
{ \overline { H } _ { 1 } ( \alpha _ { 1 } , \ldots , \alpha _ { n } ) = \beta _ { 1 } } \\
{ \overline { H } _ { 2 } ( \alpha _ { 1 } , \ldots , \alpha _ { n } ) = \beta _ { 2 } } \\
{ \vdots } \\
{ \overline { H } _ { n } ( \alpha _ { 1 } , \ldots , \alpha _ { n } ) = \beta _ { n } }
\end{array} \quad \text { or } \quad \left\{\begin{array}{c}
\bar{H}_{1}\left(\alpha_{1}, \ldots, \alpha_{n}\right)=0 \\
\bar{H}_{2}\left(\alpha_{1}, \ldots, \alpha_{n}\right)=0 \\
\vdots \\
\bar{H}_{n}\left(\alpha_{1}, \ldots, \alpha_{n}\right)=0 .
\end{array}\right.\right.
$$

Proof. By reordering the equations, we may assume that $\beta_{n} \neq 0$. Consider the $n-1$ homogeneous polynomials in $n$ variables defined by $\bar{G}_{i}=\beta_{n} \bar{H}_{i}-\beta_{i} \bar{H}_{n}$ for $i=1,2, \ldots, n-1$. By Lemma 2 , they have a nontrivial common zero 
$\left(\gamma_{1}, \ldots, \gamma_{n}\right)$. Hence

$$
\bar{H}_{i}\left(\gamma_{1}, \ldots, \gamma_{n}\right)=\frac{\bar{H}_{n}\left(\gamma_{1}, \ldots, \gamma_{n}\right)}{\beta_{n}} \beta_{i} \quad \text { for } i=1,2, \ldots, n \text {. }
$$

If $\bar{H}_{n}\left(\gamma_{1}, \ldots, \gamma_{n}\right)=0$, then $\bar{H}_{i}\left(\gamma_{1}, \ldots, \gamma_{n}\right)=0$ for all $i$. If $\bar{H}_{n}\left(\gamma_{1}, \ldots, \gamma_{n}\right) \neq$ 0 , taking $\alpha_{i}=\gamma_{i} \sqrt[d]{\frac{\beta_{n}}{\bar{H}_{n}\left(\gamma_{1}, \ldots, \gamma_{n}\right)}}$ for each $i$, we have $\bar{H}_{i}\left(\alpha_{1}, \ldots, \alpha_{n}\right)=\beta_{i}$ for all $i$.

\section{Applications}

Throughout this section, we take the convention that $\operatorname{deg} 0=0$. Note that a polynomial is not a nonzero constant means that it is either zero or of positive degree.

Lemma 4. Let $K$ be a commutative ring and let $\bar{F}_{1}, \bar{F}_{2}, \ldots, \bar{F}_{n} \in$ $K\left[x_{1}, \ldots, x_{n}\right]$ be homogeneous polynomials. If none of the $\bar{F}_{i}$ is a nonzero constant and at least one $\bar{F}_{i}$ is zero, then

$$
\operatorname{Res}_{x_{1}, \ldots, x_{n}}\left(\bar{F}_{1}, \ldots, \bar{F}_{n}\right)=0 \text {. }
$$

Proof. Without loss of generality, we may assume that $\bar{F}_{n}=0$. Since the zero polynomial is a specialization of any generic homogeneous polynomial of positive degree, there exist generic homogeneous polynomials $F_{1}\left(x_{1}, \ldots, x_{n}\right)$, $\ldots, F_{n-1}\left(x_{1}, \ldots, x_{n}\right)$ of positive degrees and a ring homomorphism

$$
\phi: \mathbb{Z}\left[\left\{\text { coefficients of } F_{1}, \ldots, F_{n-1}\right\}\right] \longrightarrow K
$$

such that $\tilde{\phi}\left(F_{i}\right)=\bar{F}_{i}$ for all $i=1,2, \ldots, n-1$, where $\tilde{\phi}$ is the natural extension of $\phi$. Note that

$$
\begin{aligned}
F_{1}, \ldots, F_{n-1} & \in \mathbb{Z}\left[\left\{\text { coefficients of } F_{1}, \ldots, F_{n-1}\right\}\right]\left[x_{1}, \ldots, x_{n}\right] \\
& \subset \mathbb{Q}\left(\left\{\text { coefficients of } F_{1}, \ldots, F_{n-1}\right\}\right)\left[x_{1}, \ldots, x_{n}\right] .
\end{aligned}
$$

Let $A$ be the field $\mathbb{Q}\left(\left\{\right.\right.$ coefficients of $\left.\left.F_{1}, \ldots, F_{n-1}\right\}\right)$, and let $L$ be its algebraic closure. By Lemma 2 , the equations $F_{1}, \ldots, F_{n-1}$ have a nontrivial solution in $L$, hence by (3) of Theorem $16, \operatorname{Res}_{x_{1}}, \ldots, x_{n}\left(F_{1}, \ldots, F_{n-1}, 0\right)=0$. Therefore, by (1) of Theorem 16 again, $\operatorname{Res}_{x_{1}, \ldots, x_{n}}\left(\bar{F}_{1}, \ldots, \bar{F}_{n}\right)=0$.

The following generalizes [13, Theorem 8, p. 357].

Corollary 5 (Chain rule for homogeneous polynomials over a commutative ring). Let $K$ be a commutative ring. Suppose for $i=1,2, \ldots, n$, that $\bar{F}_{i} \in$ $K\left[x_{1}, \ldots, x_{n}\right]$ is either zero or homogeneous of positive degree, $\bar{l}_{i}=\operatorname{deg} \bar{F}_{i}$, and suppose $\bar{H}_{i} \in K\left[u_{1}, \ldots, u_{n}\right]$ is either zero or homogeneous of positive degree d. Then

$$
\begin{aligned}
& \operatorname{Res}_{u_{1}, \ldots, u_{n}}\left(\bar{F}_{1}\left(\bar{H}_{1}, \ldots, \bar{H}_{n}\right), \ldots, \bar{F}_{n}\left(\bar{H}_{1}, \ldots, \bar{H}_{n}\right)\right) \\
& \quad=\left[\operatorname{Res}_{x_{1}, \ldots, x_{n}}\left(\bar{F}_{1}, \ldots, \bar{F}_{n}\right)\right]^{d^{n-1}}\left[\operatorname{Res}_{u_{1}, \ldots, u_{n}}\left(\bar{H}_{1}, \ldots, \bar{H}_{n}\right)\right]^{I_{1} \cdots I_{n}} .
\end{aligned}
$$

Proof. Recall the zero polynomial is a specialization of any generic homogeneous polynomial of positive degree. Let $F_{1}\left(x_{1}, \ldots, x_{n}\right), \ldots, F_{n}\left(x_{1}, \ldots, x_{n}\right)$, $H_{1}\left(u_{1}, \ldots, u_{n}\right), \ldots, H_{n}\left(u_{1}, \ldots, u_{n}\right)$ be generic homogeneous polynomials of positive degrees $l_{1}, \ldots, l_{n}, d, \ldots, d$, and let

$$
\phi: \mathbb{Z}\left[\left\{\text { coefficients of } F_{1}, \ldots, F_{n}, H_{1}, \ldots, H_{n}\right\}\right] \longrightarrow K
$$


be the ring homomorphism such that $\widetilde{\phi}\left(F_{i}\right)=\bar{F}_{i}$ and $\widetilde{\phi}\left(H_{i}\right)=\bar{H}_{i}$ for all $i$ where $\tilde{\phi}$ is the natural extension of $\phi$. Hence $l_{i}=\bar{l}_{i}$ if $\bar{F}_{i}$ is not the zero polynomial. Applying the ring homomorphism $\phi$ to both sides of equation (1) and using (1) of Theorem 16 we have that

$$
\begin{aligned}
& \operatorname{Res}_{u_{1}, \ldots, u_{n}}\left(\bar{F}_{1}\left(\bar{H}_{1}, \ldots, \bar{H}_{n}\right), \ldots, \bar{F}_{n}\left(\bar{H}_{1}, \ldots, \bar{H}_{n}\right)\right) \\
& \quad=\operatorname{Res}_{x_{1}, \ldots, x_{n}}\left(\bar{F}_{1}, \ldots, \bar{F}_{n}\right)^{d^{n-1}} \operatorname{Res}_{u_{1}, \ldots, u_{n}}\left(\bar{H}_{1}, \ldots, \bar{H}_{n}\right)^{l_{1} \cdots l_{n}} .
\end{aligned}
$$

There are two cases to consider: If $\bar{F}_{i} \neq 0$ for all $i$ then $l_{i}=\bar{l}_{i}$ for all $i$ and equation (3) holds. Otherwise $\bar{F}_{i}=0$ for some $i$. Then both

$$
\operatorname{Res}_{x_{1}, \ldots, x_{n}}\left(\bar{F}_{1}, \ldots, \bar{F}_{n}\right)
$$

and

$$
\operatorname{Res}_{u_{1}, \ldots, u_{n}}\left(\bar{F}_{1}\left(\bar{H}_{1}, \ldots, \bar{H}_{n}\right), \ldots, \bar{F}_{n}\left(\bar{H}_{1}, \ldots, \bar{H}_{n}\right)\right)
$$

are zero by Lemma 4 , and equation (3) still holds.

The following is a variation of Corollary 5 .

Corollary 6. Let $K$ be a commutative ring. Suppose for $i=1,2, \ldots, n, l_{i}$ is a positive integer, $\bar{F}_{i} \in K\left[x_{1}, \ldots, x_{n}\right]$ is either zero or homogeneous of degree $l_{i}$, and suppose $\bar{H}_{i} \in K\left[u_{1}, \ldots, u_{n}\right]$ is either zero or homogeneous of positive degree $d$. Then

$$
\begin{aligned}
& \operatorname{Res}_{u_{1}, \ldots, u_{n}}\left(\bar{F}_{1}\left(\bar{H}_{1}, \ldots, \bar{H}_{n}\right), \ldots, \bar{F}_{n}\left(\bar{H}_{1}, \ldots, \bar{H}_{n}\right)\right) \\
& =\left[\operatorname{Res}_{x_{1}, \ldots, x_{n}}\left(\bar{F}_{1}, \ldots, \bar{F}_{n}\right)\right]^{d^{n-1}}\left[\operatorname{Res}_{u_{1}, \ldots, u_{n}}\left(\bar{H}_{1}, \ldots, \bar{H}_{n}\right)\right]^{l_{1} \cdots l_{n}} .
\end{aligned}
$$

The homogenization of a polynomial $f\left(x_{1}, \ldots, x_{n-1}\right)$ with respect to $x_{n}$ is defined by

$$
f^{*}\left(x_{1}, \ldots, x_{n}\right)=x_{n}^{\operatorname{deg} f} f\left(\frac{x_{1}}{x_{n}}, \ldots, \frac{x_{n-1}}{x_{n}}\right) .
$$

If $f$ has degree $l$ then $f=f^{(0)}+f^{(1)}+\cdots+f^{(l)}$ where $f^{(i)}$ is a homogeneous polynomial of degree $i$. In this case,

$$
f^{*}=f^{(0)} x_{n}^{l}+f^{(1)} x_{n}^{l-1}+\cdots+f^{(l)} .
$$

Note that $\operatorname{deg} f^{*}=\operatorname{deg} f$ and $0^{*}=0$. Consequently, if $f$ is not a nonzero constant, neither is $f^{*}$. The resultant of $n$ polynomials in $n-1$ variables is defined to be the resultant of the corresponding homogenized polynomials.

Proposition 7. Suppose $\bar{f}_{1}, \bar{f}_{2}, \ldots, \bar{f}_{n} \in A\left[x_{1}, \ldots, x_{n-1}\right]$ where $A$ is a field with algebraic closure $L$. Then

$$
\operatorname{Res}_{x_{1}, \ldots, x_{n-1}}\left(\bar{f}_{1}, \ldots, \bar{f}_{n}\right)=0
$$

if and only if either $\bar{f}_{1}, \bar{f}_{2}, \ldots, \bar{f}_{n}$ have a common zero in $L^{n-1}$ or $\bar{f}_{1}^{+}, \bar{f}_{2}^{+}$, $\ldots, \bar{f}_{n}^{+}$have a nontrivial common zero in $L^{n-1}$.

Proof. For convenience let $R$ be $\operatorname{Res}_{x_{1}, \ldots, x_{n-1}}\left(\bar{f}_{1}, \ldots, \bar{f}_{n}\right)$. Note that, using (4) and (5), we have

$$
\begin{aligned}
& \bar{f}_{i}^{*}\left(x_{1}, \ldots, x_{n-1}, 1\right)=\bar{f}_{i}\left(x_{1}, \ldots, x_{n-1}\right), \\
& \bar{f}_{i}^{*}\left(x_{1}, \ldots, x_{n-1}, 0\right)=\bar{f}_{i}^{+}\left(x_{1}, \ldots, x_{n-1}\right) .
\end{aligned}
$$


If $\bar{f}_{1}, \bar{f}_{2}, \ldots, \bar{f}_{n}$ have common zero $\left(\beta_{1}, \ldots, \beta_{n-1}\right)$ in $L^{n}$, then, by (6), $\left(\beta_{1}, \ldots, \beta_{n-1}, 1\right)$ is a common zero for $\bar{f}_{1}^{*}, \ldots, \bar{f}_{n}^{*}$ and thus $R=0$. If $\bar{f}_{1}^{+}, \ldots, \bar{f}_{n}^{+}$have nontrivial common zero $\left(\beta_{1}, \ldots, \beta_{n-1}\right)$, then, by $(7),\left(\beta_{1}\right.$, $\left.\ldots, \beta_{n-1}, 0\right)$ is a nontrivial common zero for $\bar{f}_{1}^{*}, \ldots, \bar{f}_{n}^{*}$ and so $R=0$. This establishes sufficiency. Suppose $\bar{f}_{1}^{*}, \ldots, \bar{f}_{n}^{*}$ have a nontrivial common zero $\left(\beta_{1}, \ldots, \beta_{n}\right)$ in $L^{n}$. If $\beta_{n}=0$, then, by $(7), \bar{f}_{1}^{+}, \ldots, \bar{f}_{n}^{+}$have nontrivial common zero $\left(\beta_{1}, \ldots, \beta_{n-1}\right)$. Otherwise, by $(4), \bar{f}_{1}, \ldots, \bar{f}_{n}$ have common zero $\left(\beta_{1} / \beta_{n}, \ldots, \beta_{n-1} / \beta_{n}\right)$. Thus the necessity is established.

Lemma 8. Let $K$ be a commutative ring, and let $\bar{p}_{1}, \bar{p}_{2}, \ldots, \bar{p}_{n} \in$ $K\left[u_{1}, \ldots, u_{n-1}\right]$ be polynomials. If none of the $\bar{p}_{i}$ is a nonzero constant and at least one $\bar{p}_{i}$ is zero, then

$$
\operatorname{Res}_{u_{1}, \ldots, u_{n-1}}\left(\bar{p}_{1}, \ldots, \bar{p}_{n}\right)=0 .
$$

Proof. Without loss of generality, we may assume that $\bar{p}_{n}=0$; then $\bar{p}_{n}^{*}\left(u_{1}, \ldots, u_{n}\right)=0$, and hence

$$
\operatorname{Res}_{u_{1}, \ldots, u_{n-1}}\left(\bar{p}_{1}, \ldots, \bar{p}_{n}\right)=\operatorname{Res}_{u_{1}, \ldots, u_{n}}\left(\bar{p}_{1}^{*}, \ldots, \bar{p}_{n}^{*}\right)=0
$$

by Lemma 4 .

The following generalizes [12, Theorem 7, p. 349$]$.

Corollary 9 (Chain rule for polynomials over a commutative ring). Let $K$ be a commutative ring. Suppose for $i=1,2, \ldots, n, \bar{f}_{i} \in K\left[x_{1}, \ldots, x_{n-1}\right]$ is either zero or of positive degree, $\bar{l}_{i}=\operatorname{deg} \bar{f}_{i}$, and suppose for $j=1,2$, $\ldots, n-1, \bar{h}_{j} \in K\left[u_{1}, \ldots, u_{n-1}\right]$ is either zero or of positive degree $d$. Assume that $\operatorname{deg} \bar{f}_{i}\left(\bar{h}_{1}, \ldots, \bar{h}_{n-1}\right)=d \operatorname{deg} \bar{f}_{i}$ for all $i$, then

$$
\begin{aligned}
& \operatorname{Res}_{u_{1}, \ldots, u_{n-1}}\left(\bar{f}_{1}\left(\bar{h}_{1}, \ldots, \bar{h}_{n-1}\right), \ldots, \bar{f}_{n}\left(\bar{h}_{1}, \ldots, \bar{h}_{n-1}\right)\right) \\
& \quad=\left[\operatorname{Res}_{x_{1}, \ldots, x_{n-1}}\left(\bar{f}_{1}, \ldots, \bar{f}_{n}\right)\right]^{d^{n-1}}\left[\operatorname{Res}_{u_{1}, \ldots, u_{n-1}}\left(\bar{h}_{1}^{+}, \ldots, \bar{h}_{n-1}^{+}\right)\right]^{d \bar{I}_{1} \cdots I_{n}}
\end{aligned}
$$

where $\bar{h}_{j}^{+}$denotes the leading form of $\bar{h}_{j}$.

Proof. Let $\bar{p}_{i}\left(u_{1}, \ldots, u_{n-1}\right)=\bar{f}_{i}\left(\bar{h}_{1}, \ldots, \bar{h}_{n-1}\right)$. Then, using (4), we have

$$
\begin{aligned}
\bar{p}_{i}^{*}\left(u_{1}, \ldots, u_{n}\right) \\
=u_{n}^{\operatorname{deg} \bar{p}_{i}} \bar{p}_{i}\left(\frac{u_{1}}{u_{n}}, \ldots, \frac{u_{n-1}}{u_{n}}\right) \\
=u_{n}^{\operatorname{deg} \bar{p}_{i}} \bar{f}_{i}\left(\bar{h}_{1}\left(\frac{u_{1}}{u_{n}}, \ldots, \frac{u_{n-1}}{u_{n}}\right), \ldots \ldots, \bar{h}_{n-1}\left(\frac{u_{1}}{u_{n}}, \ldots, \frac{u_{n-1}}{u_{n}}\right)\right) \\
=u_{n}^{\operatorname{deg} \bar{p}_{i}} \bar{f}_{i}\left(\frac{\bar{h}_{1}^{*}\left(u_{1}, \ldots, u_{n}\right)}{u_{n}^{d}}, \ldots ., \frac{\bar{h}_{n-1}^{*}\left(u_{1}, \ldots, u_{n}\right)}{u_{n}^{d}}\right) \\
=u_{n}^{\operatorname{deg} \bar{p}_{i}} \frac{\bar{f}_{i}^{*}\left(\bar{h}_{1}^{*}, \ldots, \bar{h}_{n-1}^{*}, u_{n}^{d}\right)}{\left(u_{n}^{d}\right) \operatorname{deg} \bar{f}_{i}} \\
=\bar{f}_{i}^{*}\left(\bar{h}_{1}^{*}, \ldots, \bar{h}_{n-1}^{*}, u_{n}^{d}\right) .
\end{aligned}
$$


By Corollary 5, Corollary 17 and equation (5),

$$
\begin{aligned}
\operatorname{Res}_{u_{1}}, \ldots, u_{n} & \left(\bar{f}_{1}^{*}\left(\bar{h}_{1}^{*}, \ldots, \bar{h}_{n-1}^{*}, u_{n}^{d}\right), \ldots, \bar{f}_{n}^{*}\left(\bar{h}_{1}^{*}, \ldots, \bar{h}_{n-1}^{*}, u_{n}^{d}\right)\right) \\
= & {\left[\operatorname{Res}_{x_{1}, \ldots, x_{n}}\left(\bar{f}_{1}^{*}, \ldots, \bar{f}_{n}^{*}\right)\right]^{d^{n-1}}\left[\operatorname{Res}_{u_{1}, \ldots, u_{n}}\left(\bar{h}_{1}^{*}, \ldots, \bar{h}_{n-1}^{*}, u_{n}^{d}\right)\right]^{I_{1} \ldots \bar{I}_{n}} } \\
= & {\left[\operatorname{Res}_{x_{1}, \ldots, x_{n-1}}\left(\bar{f}_{1}, \ldots, \bar{f}_{n}\right)\right]^{d^{n-1}}\left[\operatorname{Res}_{u_{1}, \ldots, u_{n-1}}\left(\bar{h}_{1}^{+}, \ldots, \bar{h}_{n-1}^{+}\right)\right]^{d I_{1} \ldots \bar{I}_{n}} . }
\end{aligned}
$$

The following is a variation of Corollary 9 .

Corollary 10. Let $K$ be a commutative ring. Suppose for $i=1,2, \ldots, n$, $l_{i}$ is a positive integer, $\bar{f}_{i} \in K\left[x_{1}, \ldots, x_{n-1}\right]$ is either zero or of degree $l_{i}$, and suppose for $j=1,2, \ldots, n-1, \bar{h}_{j} \in K\left[u_{1}, \ldots, u_{n-1}\right]$ is either zero or of positive degree $d$. Assume that $\operatorname{deg} \bar{f}_{i}\left(\bar{h}_{1}, \ldots, \bar{h}_{n-1}\right)=d l_{i}$ for all $i$; then

$$
\begin{aligned}
& \operatorname{Res}_{u_{1}, \ldots, u_{n-1}}\left(\bar{f}_{1}\left(\bar{h}_{1}, \ldots, \bar{h}_{n-1}\right), \ldots, \bar{f}_{n}\left(\bar{h}_{1}, \ldots, \bar{h}_{n-1}\right)\right) \\
& \quad=\left[\operatorname{Res}_{x_{1}, \ldots, x_{n-1}}\left(\bar{f}_{1}, \ldots, \bar{f}_{n}\right)\right]^{d^{n-1}}\left[\operatorname{Res}_{u_{1}, \ldots, u_{n-1}}\left(\bar{h}_{1}^{+}, \ldots, \bar{h}_{n-1}^{+}\right)\right]^{d l_{1} \cdots l_{n}}
\end{aligned}
$$

where $\bar{h}_{j}^{+}$denotes the leading form of $\bar{h}_{j}$.

The proof of the following is similar and therefore will be omitted.

Corollary 11 (Chain rule for generic polynomials). Suppose $f_{i}\left(x_{1}, \ldots, x_{n-1}\right)$ and $h_{i}\left(u_{1}, \ldots, u_{n-1}\right)$ are generic polynomials of positive degrees $l_{i}$ and $d$ respectively for $i=1,2, \ldots, n$. Then

$$
\begin{aligned}
& \operatorname{Res}_{u_{1}, \ldots, u_{n-1}}\left(f_{1}\left(h_{1}, \ldots, h_{n-1}\right), \ldots, f_{n}\left(h_{1}, \ldots, h_{n-1}\right)\right) \\
& \quad=\left[\operatorname{Res}_{x_{1}, \ldots, x_{n-1}}\left(f_{1}, \ldots, f_{n}\right)\right]^{d^{n-1}}\left[\operatorname{Res}_{u_{1}, \ldots, u_{n-1}}\left(h_{1}^{+}, \ldots, h_{n-1}^{+}\right)\right]^{d l_{1} \cdots l_{n}}
\end{aligned}
$$

where $h_{j}^{+}$denotes the leading form of $h_{j}$.

The discriminant $\operatorname{Disc}(F)$ of a homogeneous generic polynomial $F\left(x_{1}, \ldots, x_{n}\right)$ is the resultant of its partial derivatives $\frac{\partial F}{\partial x_{1}}, \ldots, \frac{\partial F}{\partial x_{n}}$. The following generalizes [2, Corollary, p. 98].

Corollary 12. Suppose $F\left(x_{1}, \ldots, x_{n}\right)$ and $H_{i}\left(u_{1}, \ldots, u_{n}\right)$, for $i=1,2$, $\ldots, n$, are generic homogeneous polynomials of degrees at least two. Then

$$
\operatorname{Disc}\left(F\left(H_{1}, \ldots, H_{n}\right)\right)=[\operatorname{Disc}(F)]^{m}\left[\operatorname{Res}_{u_{1}, \ldots, u_{n}}\left(H_{1}, \ldots, H_{n}\right)\right]^{k} G
$$

for some positive integers $m$ and $k$, and some $G$ in the polynomial ring $\mathbb{Q}\left[\left\{\right.\right.$ coefficients of $\left.\left.F, H_{1}, \ldots, H_{n}\right\}\right]$.

Proof. Let $P\left(u_{1}, \ldots, u_{n}\right)=F\left(H_{1}, \ldots, H_{n}\right)$. By the chain rule for partial derivatives, we have

$$
\begin{aligned}
& {\left[\begin{array}{lll}
\frac{\partial P}{\partial u_{1}} & \cdots & \frac{\partial P}{\partial u_{n}}
\end{array}\right]} \\
& =\left[\begin{array}{lll}
\frac{\partial F}{\partial x_{1}}\left(H_{1}, \ldots, H_{n}\right) & \ldots & \frac{\partial F}{\partial x_{n}}\left(H_{1}, \ldots, H_{n}\right)
\end{array}\right]\left[\begin{array}{lll}
\frac{\partial H_{1}}{\partial u_{1}} & \ldots & \frac{\partial H_{1}}{\partial u_{n}} \\
\dddot{\partial} \ldots \ldots & \ldots . & \ldots \\
\frac{\partial H_{n}}{\partial u_{1}} & \ldots & \frac{\partial H_{n}}{\partial u_{n}}
\end{array}\right] .
\end{aligned}
$$

It follows that $\frac{\partial P}{\partial u_{1}}, \ldots, \frac{\partial P}{\partial u_{n}}$ have a nontrivial common zero whenever $\frac{\partial F}{\partial x_{1}}\left(H_{1}, \ldots, H_{n}\right), \ldots, \frac{\partial F}{\partial x_{n}}\left(H_{1}, \ldots, H_{n}\right)$ have a nontrivial common zero. By 
(3) of Theorem 16, this amounts to saying that $\operatorname{Res}_{u_{1}, \ldots, u_{n}}\left(\frac{\partial P}{\partial u_{1}}, \ldots, \frac{\partial P}{\partial u_{n}}\right)$ vanishes whenever $\operatorname{Res}_{u_{1}, \ldots, u_{n}}\left(\frac{\partial F}{\partial x_{1}}\left(H_{1}, \ldots, H_{n}\right), \ldots, \frac{\partial F}{\partial x_{n}}\left(H_{1}, \ldots, H_{n}\right)\right)$ vanishes. Hence by Hilbert's Nullstellensatz, in $\mathbb{Q}\left[\left\{\right.\right.$ coefficients of $\left.\left.F, H_{1}, \ldots, H_{n}\right\}\right]$, every irreducible factor of $\operatorname{Res}_{u_{1}, \ldots, u_{n}}\left(\frac{\partial F}{\partial x_{1}}\left(H_{1}, \ldots, H_{n}\right), \ldots, \frac{\partial F}{\partial x_{n}}\left(H_{1}, \ldots, H_{n}\right)\right)$ is also a factor of $\operatorname{Res}_{u_{1}}, \ldots, u_{n}\left(\frac{\partial P}{\partial u_{1}}, \ldots, \frac{\partial P}{\partial u_{n}}\right)$. Using property (2) of Theorem 16 below and the fact that the discriminant of a generic polynomial is irreducible (see, for example, [6]), we see that $\operatorname{Res}_{u_{1}, \ldots, u_{n}}\left(H_{1}, \ldots, H_{n}\right)$ and $\operatorname{Disc}(F)$ are irreducible. Thus these are the only irreducible factors of $\operatorname{Res}_{u_{1}, \ldots, u_{n}}\left(\frac{\partial F}{\partial x_{1}}\left(H_{1}, \ldots, H_{n}\right), \ldots, \frac{\partial F}{\partial x_{n}}\left(H_{1}, \ldots, H_{n}\right)\right)$ by Theorem 1 and the result follows.

The following two properties were deduced by Macaulay [10, pp. 11-12] from his construction of the resultant. We shall deduce them from Theorem 1 without using the actual construction.

Corollary 13 (Invariant Property). Let $M=\left(m_{i j}\right)_{j=1, \ldots, n}^{i=1, \ldots, n}$ be a matrix over a commutative ring $K$, and let

$$
C_{k}\left(u_{1}, \ldots, u_{n}\right)=F_{k}\left(\sum_{j=1}^{n} m_{1 j} u_{j}, \ldots, \sum_{j=1}^{n} m_{n j} u_{j}\right)
$$

where $F_{k} \in K\left[x_{1}, \ldots, x_{n}\right]$ is either zero or homogeneous of positive degree $l_{k}$, $k=1,2, \ldots, n$. Then

$$
\operatorname{Res}_{u_{1}, \ldots, u_{n}}\left(C_{1}, \ldots, C_{n}\right)=(\operatorname{det} M)^{l_{1} \cdots l_{n}} \operatorname{Res}_{x_{1}, \ldots, x_{n}}\left(F_{1}, \ldots, F_{n}\right) .
$$

Proof. Let $H_{i}=\sum_{j=1}^{n} m_{i j} u_{j}$. Then $\operatorname{Res}_{u_{1}, \ldots, u_{n}}\left(H_{1}, \ldots, H_{n}\right)=\operatorname{det} M$ since $\operatorname{det} M$ satisfies all four conditions of Theorem 16, and the result follows from Corollary 6 by taking $d=1$.

Corollary 14 (Isobaric Property). Suppose $F_{i}\left(x_{1}, \ldots, x_{n}\right)$ is a generic homogeneous polynomial of positive degree $l_{i}$ for $i=1,2, \ldots, n$. Then

$$
\begin{aligned}
& \operatorname{Res}_{x_{1}, \ldots, x_{n}}\left(F_{1}\left(x_{1}, \ldots, t x_{k}, \ldots, x_{n}\right), \ldots, F_{n}\left(x_{1}, \ldots, t x_{k} \ldots, x_{n}\right)\right) \\
& \quad=t^{l_{1} \cdots l_{n}} \operatorname{Res}_{x_{1}, \ldots, x_{n}}\left(F_{1}, \ldots, F_{n}\right)
\end{aligned}
$$

for $k=1,2, \ldots, n$. In other words, with respective to any variable $x_{k}$, the resultant is isobaric of weight $l_{1} \cdots l_{n}$.

Proof. Take $m_{i j}=\delta_{i j}$ when $(i, j) \neq(k, k), m_{k k}=t$, and

$$
K=\mathbb{Z}\left[\left\{\text { coefficients of } K_{1}, \ldots, K_{n}\right\}, t\right] \text {. }
$$

Then this follows immediately from Corollary 13.

Corollary 15. Let $M=\left(m_{i j}\right)_{j=1, \ldots, n}^{i=1, \ldots, n}$ be a matrix over a commutative ring $K$. If $H_{1}, \ldots, H_{n} \in K\left[u_{1}, \ldots, u_{n}\right]$ are homogeneous of positive degree $d$ then

$$
\begin{array}{r}
\operatorname{Res}_{u_{1}, \ldots, u_{n}}\left(\sum_{j=1}^{n} m_{1 j} H_{j}, \ldots, \sum_{j=1}^{n} m_{n j} H_{j}\right) \\
=(\operatorname{det} M)^{d^{n-1}} \operatorname{Res}_{u_{1}, \ldots, u_{n}}\left(H_{1}, \ldots, H_{n}\right) .
\end{array}
$$

Proof. Take $F_{i}\left(x_{1}, \ldots, x_{n}\right)=\sum_{j=1}^{n} m_{i j} x_{j}$, and apply Corollary 6 . 


\section{AXIOMATIC CHARACTERIZATION OF RESUltANTS}

In 1903, Macaulay $[9, \S 6$, Theorem, p. 14] discovered an explicit formula expressing the multivariable resultant as the quotient of two determinants (see also [10, pp. 3-15]). The next theorem is taken, with a slight modification, from Chardin's 1990 thesis [3, Théorème, p. 37], where he clearly enunciates the defining properties of the resultant (see also [5, Theorem 3.1]). He said, in a private communication, 'It is a direct consequence of the so-called "Fundamental theorem of elimination theory" which states that the elimination ideal is principal and behaves well when you change the base ring ; these are quite old results, I found them in an unpublished work of Bourbaki but also in Philippon or Jouanolou.'

Theorem 16. For every commutative ring $A$, and every $n$-tuple, $n>0,\left(P_{1}\right.$, $\left.\ldots, P_{n}\right)$ of homogeneous polynomials of degrees $l_{1}, \ldots, l_{n}$ in $A\left[x_{1}, \ldots, x_{n}\right]$ such that no $P_{i}$ is a nonzero constant, there exists an element $\operatorname{Res}_{x_{1}, \ldots, x_{n}}\left(P_{1}\right.$, $\left.\ldots, P_{n}\right)$ of $A$ which is uniquely determined by the following four properties.

(1) If $\phi: A \longrightarrow B$ is a ring homomorphism and $\widetilde{\phi}: A\left[x_{1}, \ldots, x_{n}\right] \longrightarrow$ $B\left[x_{1}, \ldots, x_{n}\right]$ its natural extension, then

$$
\operatorname{Res}_{x_{1}, \ldots, x_{n}}\left(\tilde{\phi}\left(P_{1}\right), \ldots, \tilde{\phi}\left(P_{n}\right)\right)=\phi\left(\operatorname{Res}_{x_{1}, \ldots, x_{n}}\left(P_{1}, \ldots, P_{n}\right)\right) \text {. }
$$

(2) If $P_{1}, \ldots, P_{n}$ are generic homogeneous polynomials of positive degrees, then $\operatorname{Res}_{x_{1}, \ldots, x_{n}}\left(P_{1}, \ldots, P_{n}\right)$ is irreducible in the polynomial ring

$$
\mathbb{Z}\left[\left\{\text { coefficients of } P_{1}, \ldots, P_{n}\right\}\right] \text {. }
$$

(3) If $A$ is a field and $L$ its algebraic closure, then $\operatorname{Res}_{x_{1}, \ldots, x_{n}}\left(P_{1}, \ldots, P_{n}\right)$ is zero if and only if $P_{1}, \ldots, P_{n}$ have a nontrivial common zero in $L^{n}$.

(4) $\operatorname{Res}_{x_{1}, \ldots, x_{n}}\left(a_{1} x_{1}^{l_{1}}, \ldots, a_{n} x_{n}^{l_{n}}\right)=a_{1}^{l_{2} \cdots l_{n}} a_{2}^{l_{1} l_{3} \cdots l_{n}} \ldots a_{n}^{l_{1} \cdots l_{n-1}}$ for $a_{i} \in A$ and positive integer $l_{i}$.

Proof. Existence. For $n=1$, define the resultant of a homogeneous polynomial $a x_{1}^{l_{1}}$ to be $a$. For $n>1$, Macaulay [10] has defined the resultant $\operatorname{Res}_{x_{1}, \ldots, x_{n}}\left(P_{1}, \ldots, P_{n}\right)$ for generic homogeneous polynomials $P_{1}, \ldots, P_{n}$ of positive degrees in variables $x_{1}, \ldots, x_{n}$, i.e., when

$$
\left.A=\mathbb{Z}\left[\text { coefficients of } P_{1}, \ldots, P_{n}\right\}\right] .
$$

In general, if $P_{i} \in A\left[x_{1}, \ldots, x_{n}\right]$ is homogeneous and not a nonzero constant then one may choose generic homogeneous polynomial $\widetilde{P}_{i}$ with $\operatorname{deg} \widetilde{P}_{i}=\operatorname{deg} P_{i}$ such that $\widetilde{\phi}\left(\widetilde{P}_{i}\right)=P_{i}$ for all $i$ where $\widetilde{\phi}$ is the obvious extension of some ring homomorphism $\phi: \mathbb{Z}\left[\left\{\right.\right.$ coefficients of $\left.\left.P_{1}, \ldots, P_{n}\right\}\right] \longrightarrow A$. Then the resultant of $P_{1}, \ldots, P_{n}$ in $A$ is defined to be $\phi\left(\operatorname{Res}_{x_{1}}, \ldots, x_{n}\left(\widetilde{P}_{1}, \ldots, \widetilde{P}_{n}\right)\right)$, and so (1) follows immediately. Both (2) and (3) are proved in [10, I.9, p. 12 and I.10, p. 13]. Note that, from [10, I.7, p. 8], if each $P_{i}$ is a generic homogeneous polynomial of positive degree then $\operatorname{Res}\left(P_{1}, \ldots, P_{n}\right)$ is homogeneous in coefficients of $P_{i}$ of degree $l_{1} \cdots l_{n} / l_{i}$. Thus (4) follows from [10, I.8, p. 10] and (1).

Uniqueness. It is enough to establish the case where

$$
A=\mathbb{Z}\left[\left\{\text { coefficients of } P_{1}, \ldots, P_{n}\right\}\right]
$$

because the general case follows by applying (1). Suppose both $R^{\prime}=R^{\prime}\left(P_{1}\right.$, $\left.\ldots, P_{n}\right)$ and $R=R\left(P_{1}, \ldots, P_{n}\right)$ satisfy all four conditions where $P_{1}, \ldots, P_{n}$ 
are generic homogeneous polynomials, none of which is a nonzero constant. Using (2), (3) and the Nullstellensatz, we see that $R$ and $R^{\prime}$ differ by a constant multiple in $\mathbb{Q}$. This constant must be 1 by (4).

Remark. The above theorem is not true if the condition "no $P_{i}$ is a nonzero constant" is removed since $\operatorname{Res}\left(f_{1}, f_{2}\right)$ does not exist when $f_{1}=a_{1}$ and $f_{2}=a_{2}$ are generic homogeneous of degree 0 . For $\operatorname{Res}\left(f_{1}, f_{2}\right)$ is in $\mathbb{Z}[$ coefficients of $\left.f_{1}, f_{2}\right\}$ ] and thus is a polynomial $f\left(a_{1}, a_{2}\right)$ in $a_{1}$ and $a_{2}$ over $\mathbb{Z}$, and using (1) and (3) of Theorem 16, we see that, for every ring homomorphism $\phi: \mathbb{Z}\left[a_{1}, a_{2}\right] \longrightarrow \mathbb{C}$,

$$
\operatorname{Res}\left(\phi\left(a_{1}\right), \phi\left(a_{2}\right)\right)=0 \Longleftrightarrow \phi\left(a_{1}\right)=\phi\left(a_{2}\right)=0 .
$$

This is equivalent to saying that $f\left(a_{1}, a_{2}\right)$ has only trivial zeros. But this is impossible by Lemma 2 .

Corollary 17. Let $A$ be a commutative ring and $P_{i} \in A\left[x_{1}, \ldots, x_{n}\right], i=1,2$, $\ldots, n$, be homogeneous such that none is a nonzero constant. Then

$$
\operatorname{Res}_{x_{1}, \ldots, x_{n}}\left(P_{1}, \ldots, P_{n-1}, x_{n}^{d}\right)=\operatorname{Res}_{x_{1}, \ldots, x_{n-1}}\left(P_{1}\left[x_{n}=0\right], \ldots, P_{n-1}\left[x_{n}=0\right]\right)^{d} \text {. }
$$

Proof. This follows immediately from (3) of Theorem 16.

Addendum. After the manuscript was accepted for publication, we were informed that some of our results were obtained independently by J. P. Jouanolou using different methods.

\section{REFERENCES}

1. N. Bourbaki, Commutative algebra, Elements of Mathematics, Addison-Wesley, Reading, MA, 1972.

2. F. Faà de Bruno, Théorie des formes binaires, Résumé des leçons faites à l'Université de Turin, Librairie Brero succ ${ }^{\mathrm{r}}$. de P. Marietti, Turin, 1876.

3. M. Chardin, Contributions à l'algèbre commutative effective et à la théorie de l'élimination, Thèse de l'Université Pierre et Marie Curie (Paris VI), 1990, preprint of Centre de Mathématiques de l'Ecole Polytechnique F-91128 Palaiseau France.

4. C. C. Cheng, J. H. McKay, and S. S.-S. Wang, Chain rule for multivariable resultants, Abstracts Amer. Math. Soc. 13 (1992), 242 (873-13-109).

5. A. van den Essen and M. Kwieciński, On the reconstruction of polynomial automorphisms from their face polynomials, J. Pure Appl. Algebra 80 (1992), 327-336.

6. I. M. Gel'fand, A. V. Zelevinskii, and M. M. Kapranov, Discriminants of polynomials in many variables, Funct. Anal. Appl. 24 (1990), 1-4.

7. N. Kravitsky and Z. Waksman, On some resultant identities, Linear Algebra Appl. 122-124 (1989), 3-21.

8. E. Kunz, Introduction to commutative algebra and algebraic geometry, Einführung in die kommutative Algebra und algebraische Geometrie, Birkhäuser, Boston, MA, 1985.

9. F. S. Macaulay, Some formula in elimination, Proc. London Math. Soc. 35 (1903), 3-27.

10. __ The algebraic theory of modular systems, Cambridge Tracts in Math., vol. 19, Cambridge Univ. Press, Cambridge, 1916.

11. J. H. McKay and S. S.-S. Wang, Chain rule for resultant, Abstracts Amer. Math. Soc. 8 (1987), 329 (836-13-122).

12. _ A chain rule for the resultant of two polynomials, Arch. Math. 53 (1989), 347-351. 
13. A A chain rule for the resultant of two homogeneous polynomials, Arch. Math. 56 (1991), 352-361.

14. P. Philippon, Critères pour l'indépendance algébrique, Inst. Hautes Études Sci. Publ. Mat. 64 (1986), 5-52.

Department of Mathematical Sciences, Oakland University, Rochester, Michigan 48309-4401

E-mail address, C. C.-A. Cheng: cheng@vela.acs.oakland.edu

E-mail address, J. H. McKay: mckay@vela.acs.oakland.edu

E-mail address, S. S.-S. Wang: swang@vela.acs.oakland.edu 